\title{
Reversal of particle-hole scattering-rate asymmetry in Anderson impurity model
}

\author{
R. Žitko, ${ }^{1,2}$ H. R. Krishnamurthy, ${ }^{3}$ and B. Sriram Shastry ${ }^{4}$ \\ ${ }^{1}$ Jožef Stefan Institute, Jamova 39, SI-1000 Ljubljana, Slovenia \\ ${ }^{2}$ Faculty of Mathematics and Physics, University of Ljubljana, Jadranska 19, SI-1000 Ljubljana, Slovenia \\ ${ }^{3}$ Department of Physics, Center for Condensed Matter Theory, \\ Indian Institute of Science, Bengaluru - 560012, India \\ ${ }^{4}$ Physics Department, University of California, Santa Cruz, CA 95064
}

(Dated: October 11, 2018)

\begin{abstract}
We study the particle-hole asymmetry of the scattering rate in strongly correlated electron systems by examining the cubic $\omega^{3}$ and $\omega T^{2}$ terms in the imaginary part of the self-energy of the Anderson impurity model. We show that the sign is opposite in the weak-coupling and strong-coupling limits, explaining the differences found in theoretical approaches taking the respective limits as the starting points. The sign change in fact precisely delineates the cross-over between the weak and strong correlation regimes of the model. For weak interaction $U$ the sign reversal occurs for small values of the doping $\delta=1-n$, while for interaction of order $U \approx 2 \Gamma$, $\Gamma$ being the hybridization strength, the cross-over curve rapidly shifts to the large-doping range. This curve based on the impurity dynamics is genuinely different from other cross-over curves defined through impurity thermodynamic and static properties.
\end{abstract}

In contemporary strongly correlated quantum materials, such as the cuprate superconductors and sodium cobaltates, one finds that spectral line shapes from angle-resolved photoemission spectroscopy (ARPES) differ qualitatively from those in simple Fermi liquids. The origin of the difference has been traced to a large correlation-induced asymmetry in the imaginary part of the self-energy ${ }^{112}$, which can be expanded as

$$
\operatorname{Im} \Sigma(\omega, T)=a\left(\omega^{2}+\pi^{2} T^{2}\right)+b \omega^{3}+c \omega T^{2}+\ldots
$$

For context note that the usually quoted Fermi-liquid selfenergy, namely the first two terms in this expression, is even in $\omega$. While this is dominant at the lowest energies, the higher order odd in $\omega$ terms become important when their coefficients $(b, c)$ become sufficiently large. This is found to happen in the strong-correlation models, while in weakly correlated systems these coefficients are very small. The signs of $(b, c)$ are of particular importance: they determine whether particles or holes have the shorter lifetime. Since $a<0$, if $b<0$ the particle-like excitations scatter more strongly on the impurity (are more damped) than the hole-like excitations with the same excitation energy (absolute value of $\omega$ ), and vice-versa for $b>0$. Understanding the asymmetry of the self-energy is a problem of great current interest. The asymmetry of $\operatorname{Im} \Sigma$ is relevant to transport coefficients such as the thermopower, where the entropy and charge are carried by both particle-like excitations above the Fermi level and hole-like excitations below it. However in the low- $T$ thermopower there are other competing factors (asymmetry of the density of states, asymmetry of the quasiparticle velocities), hence the situation is not solely controlled by the sign of the scattering asymmetry.

The single impurity Anderson model (SIAM) is a "laboratory example" of an exactly solvable many-body problem. It is simpler but has many similarities to the lattice many-body problems such as the Hubbard model. It is therefore a natural place to understand the magnitude and signs of the asymmetric corrections to the lowest-order Fermi liquid theory result mentioned above. The goal of this paper is to explore this asymmetry by using the numerical renormalization group (NRG), and to contrast it with various approximate theories.
We report a surprising result in this well-studied problem: we find a line in the $U-n$ plane where the asymmetry changes sign. Here $U$ is the interaction strength and $n$ the impurity occupancy. Along this 1-dimensional line in $U-n$ plane, the particle-hole symmetry of the scattering rate is exactly fulfilled up to the fifth and higher order terms. This change of sign demarcates the border between the qualitatively different regimes of weak and strong correlations. Indeed, this work was motivated by the puzzling observation that weakcoupling approaches (e.g., perturbation theory in the interaction strength $U$ ) and strong-coupling techniques (e.g., the extremely correlated Fermi liquid (ECFL) theory ${ }^{2 / 4}$ ) give opposite signs for the asymmetry, as illustrated in Fig. 1.

The SIAM is defined by the Hamiltonian

$$
H=\epsilon_{d} n+U n_{\uparrow} n_{\downarrow}+\sum_{k \sigma} \epsilon_{k} c_{k \sigma}^{\dagger} c_{k \sigma}+\sum_{k \sigma}\left(t_{k} c_{k \sigma}^{\dagger} d_{\sigma}+\text { h.c. }\right)
$$

where $\epsilon_{d}$ is the impurity level, $d_{\sigma}$ are impurity operators, $n_{\sigma}=d_{\sigma}^{\dagger} d_{\sigma}$ and $n=n_{\uparrow}+n_{\downarrow}, c_{k \sigma}$ are operators for conduction-band electrons with energy $\epsilon_{k}$ that couple with the impurity with amplitude $t_{k}$. The hybridisation strength is $\Gamma=\pi \sum_{k}\left|t_{k}\right|^{2} \delta\left(\omega-\epsilon_{k}\right)$; we will assume it to be a constant function in the domain $-D<\omega<D$. In the following we will make use of the Hartree-Fock parameter $E_{d}=\epsilon_{d}+U\left\langle n_{\sigma}\right\rangle$ with $E_{d}=0$ corresponding to the p-h symmetric $n=1$ case. We also define the dimensionless interaction $u=U / \pi \Gamma$. We will limit our consideration to $n<1$, since the results for $n>1$ can be obtained by the $\mathrm{p}-\mathrm{h}$ transformation $d_{\sigma} \rightarrow d_{\sigma}^{\dagger}, c_{k \sigma} \rightarrow-c_{k \sigma}^{\dagger}$ which takes $\omega$ to $-\omega$.

Second-order perturbation theory in $U$ for the $\operatorname{SIAM}^{5}$ (e.g. Fig. 1 (a) for $E_{d} / \Gamma=1$ ) predicts $b$ to be of constant sign as a function of $n$ in the full domain $0<n<1$, with zero value at $n=1$. Specifically, $b<0$, i.e. the particle-like excitations scatter more strongly. At non-zero but low temperatures, the asymmetry of $\operatorname{Im} \Sigma(\omega)$ at low $\omega$ will be controlled by the $\omega T^{2}$ term. Due to conformal symmetry of the FL fixed point, $c=$ $b \pi^{2}$, thus the sign of the asymmetry does not depend on $\mathrm{T}$ at low enough temperatures ${ }^{6}$. 


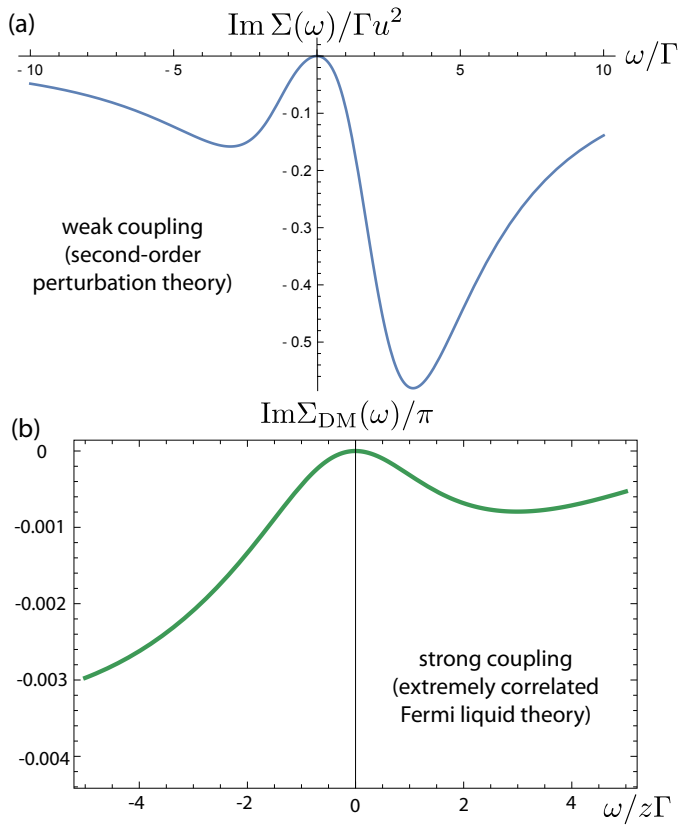

Figure 1. Imaginary parts of the self-energy in (a) weak-coupling theory and in (b) strong-coupling theory have the opposite sign of the particle-hole asymmetry. The second-order perturbation theory result corresponds to $E_{d} / \Gamma=1$ in Fig. 2 of Ref. 5, the extremelycorrelated Fermi liquid theory result to $n=0.6$ in Fig. 5 of Ref. 4

By extending the perturbative expansion to third order, we find that the third-order contributions tend to have the opposite sign of the second-order ones for small $\omega$, i.e., they reduce the scattering. Furthermore, the asymmetry of the thirdorder contribution is such that the $\omega>0$ part is dominant (for $n<1$ ), thus the third-order reduction in scattering is stronger for particle-like excitations, see Fig. 2(a). There is thus a competition between the second and third-order contributions which may lead to a change in sign of the asymmetric terms. The contributions $b^{(2)}$ and $b^{(3)}$ to the coefficient $b$ actually follow very similar qualitative $E_{d}$ dependence, see Fig. 2(b), except for the sign and evidently a different power of $u=U / \pi \Gamma$. Based on these results, the change of sign in $b$ should occur for $u \sim 1$, i.e., $U \sim \pi \Gamma$. Since the perturbation theory also breaks down at $u \sim 1$, we cannot make any more precise statement about the details of this sign change within the bare perturbative approach.

We therefore solved the impurity problem numerically using the numerical renormalization group (NRG) $)^{7 \mid 12}$. This non-perturbative approach is based on logarithmic discretization of the continuum, mapping onto a tight-binding chain with exponentially decreasing hopping constants, and iterative diagonalization of the resulting Hamiltonian $7 / 8 / 12$. Through various refinements over the years $\frac{11 \mid 13}{20}$ the technique has developed into a powerful tool for computing dynamical properties of impurity models. Comparisons with quantum Monte Carlo simulations indicate that the results of the NRG, when taken to full convergence, may be considered as essentially exact (up to very small systematic errors due to discretization and truncation). We performed the NRG calculations
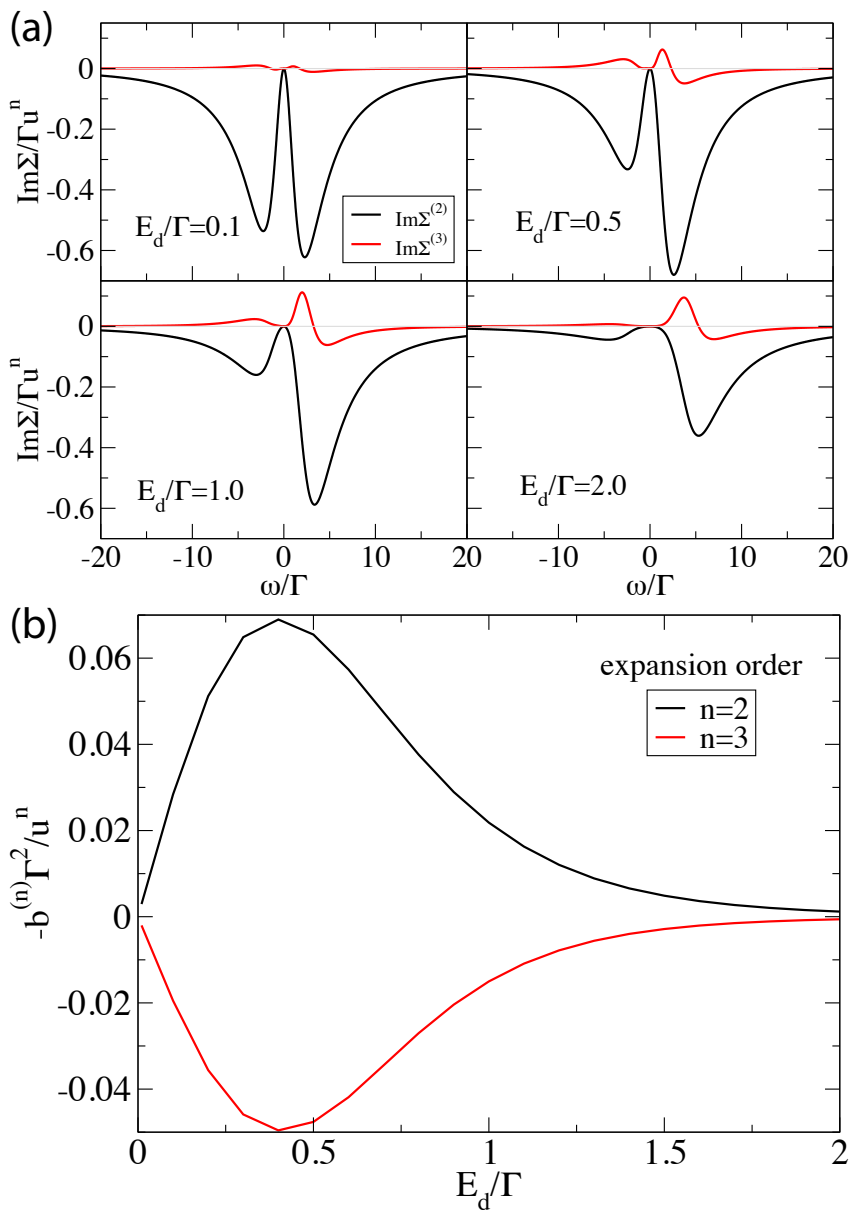

Figure 2. (a) Second-order and third-order imaginary part of the selfenergy for a range of the Hartree-Fock parameters $E_{d}=\epsilon_{d}+U\left\langle n_{\sigma}\right\rangle$. The $y$-axis is scaled as $1 /\left(\Gamma u^{n}\right)$ where $n$ is the expansion order and $u=U / \pi \Gamma . T=0$. (b) Coefficient $b$ of the $\omega^{3}$ term in $\operatorname{Im} \Sigma(\omega, T=$ $0)$ at second and third order in perturbation theory.

with a narrow broadening kernel by averaging over $N_{z}=$ 32 interleaved discretization grids with the discretization parameter $\Lambda=2$, and increasing the truncation cutoff until convergence $^{20}$ : these steps reduced the oscillatory artifacts and allowed a reliable extraction of the cubic term in the selfenergy function in the limit of small $\omega$. The results, shown in Fig. 3, reveal a change of sign of the coefficient $b$ in $\operatorname{Im} \Sigma$ along a curve in the $U-n$ plane (black line in the figure). At low $U \ll \Gamma$, the sign reversal occurs close to half-filling. For $U$ of order $\Gamma$, the sign-change point rapidly moves away from half-filling. At still higher $U$, the slope of the black curve in the $U-n$ plane redresses and becomes increasingly vertical for $U \gg \pi \Gamma$.

These results are fully consistent with our perturbative analysis. For very small $U$, the third order term is negligibly small and the sign is constant in essentially the full $0<n<1$ interval. In this regime, the curve separating the different signs in the $U-n$ plane is almost vertical and close to $n=1$. At some value of $U$ of order $\pi \Gamma$, the perturbation theory predicts that the third-order contribution will overtake the second- 
order contribution for most $E_{d}$ at almost the same value of $u$, and $b$ will thus change in a wide $n$ interval. Indeed, this seems to correspond to $U \approx 2 \Gamma$ where the curve in the $U-n$ plane abruptly changes slope and becomes almost horizontal. Around the same $u$, however, we enter the strong-coupling regime where the perturbation theory breaks down. Note that for large $u=U / \Gamma$ the crossover (as $n$ increases from 0 ) to the "strong coupling" domain with $b>0$ already takes place in the mixed valent regime of the impurity model; the deep Kondo limit is confined to values of $n$ close to 1 .

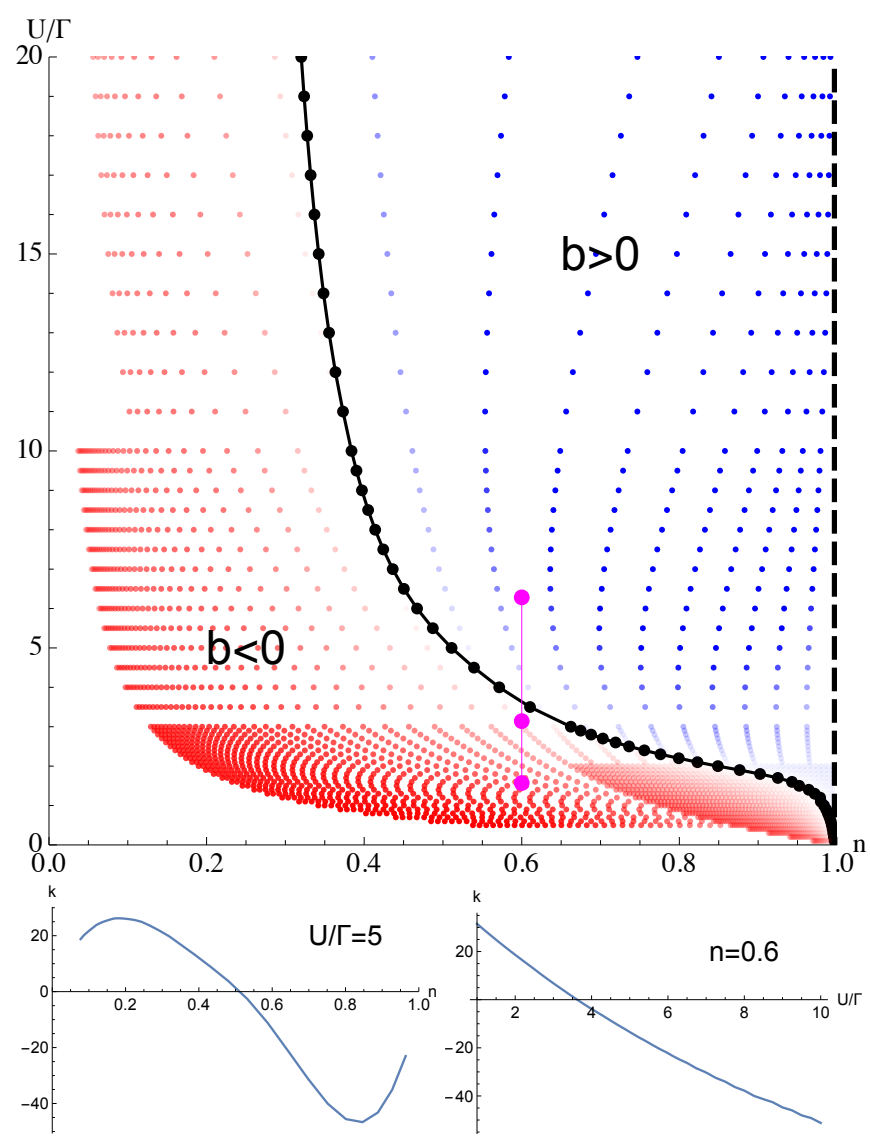

Figure 3. Top: Sign (red negative, blue positive) and magnitude (color saturation) of the $\omega^{3}$ term in $\operatorname{Im} \Sigma(\omega, T=0)$. The quantity shown is the coefficient $k$ in the fit of the antisymmetrized and normalized combination $[\operatorname{Im} \Sigma(\omega)-\operatorname{Im} \Sigma(-\omega)] /[\operatorname{Im} \Sigma(\omega)+\operatorname{Im} \Sigma(-\omega)]$ with the linear function $k \omega$. The fit is performed in an energy interval $\omega \in[-\xi: \xi]$; here $\xi$ is the low-energy scale of the problem defined as the temperature where the impurity moment is screened (and is equivalent to the Kondo temperature in the Kondo regime of the model). Notice that $k \approx b / a$ and that $a<0$. The three magenta points joined by a line are considered in Fig. 4 The dashed lined at $n=1$ indicates a further zero-crossing of the coefficient $k$ at the particle-hole symmetric point of the Hamiltonian itself. Bottom: cross-sections at constant interaction $U / \Gamma=5$ and constant occupancy $n=0.6$.

We also performed the skeleton expansion to second order, which is a self-consistent calculation where the dressed Green's function is used as the propagator in the second-order term of the self-energy. This corresponds to an infinite re- sumation of a certain class of diagrams, which for small $U$ reduces to the bare perturbation theory in $U$. The results show that the coefficient $b$ has the same sign for any value of $U$ and $n$, i.e., the sign associated with the weak-coupling limit, see Fig. 4. This can also be shown analytically by invoking the Friedel sum rule 21 - for the skeleton expansion this leads to $b / a \propto \sin (n \pi) / \Gamma$ which makes $b$ negative for all $n$. The absence of sign change seems to imply that the skeleton expansion is not able to describe the transition to the dynamics expected in the strong-coupling regime, presumably because its starting point is still the non-interacting limit. On the other hand, the extremely correlated Fermi liquid theory produces the correct sign of the scattering-rate asymmetry because it is constructed as a strong-coupling approach by projecting out the double-occupancy from the outset.
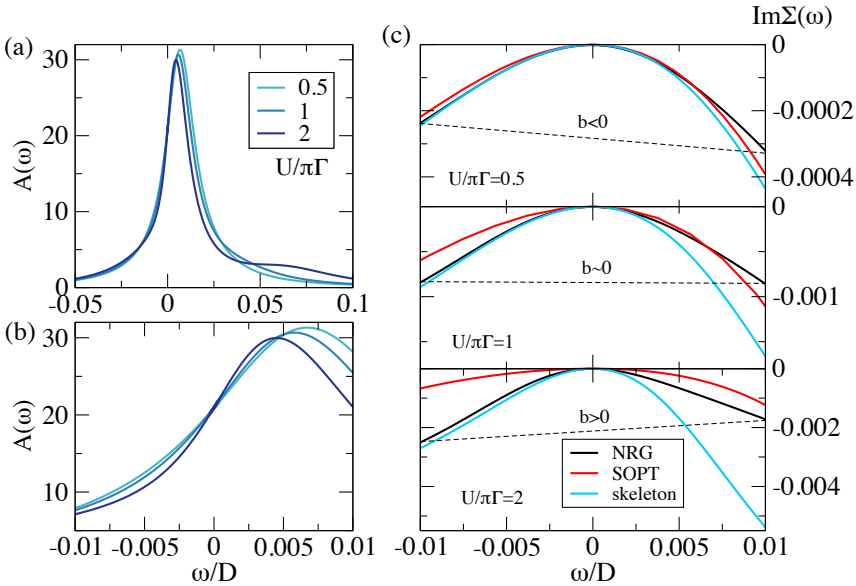

Figure 4. Spectral function and imaginary part of self-energy for $n=$ 0.6 and three values of $U / \pi \Gamma$ across the sign-reversal line (magenta line in Fig. 3). (a) Spectral functions computed using the NRG, and (b) close-ups on the low- $\omega$ range. (c) $\operatorname{Im} \Sigma$ computed using the NRG and two weak-coupling approaches. Here $\Gamma=0.01 D, T=0 . b$ for the NRG calculations has the sign of the slopes of the dashed lines in the figures.

Next, we compare the cross-over curve with other quantities sensitive to the magnetic behavior of the impurity. The local-moment fraction $f_{\mathrm{LM}}=\langle n\rangle-2\left\langle n_{\uparrow} n_{\downarrow}\right\rangle=2\langle n\rangle-\left\langle n^{2}\right\rangle$ is equal to the expectation value of the projection operator to the singly-occupied impurity state (the state which carries the spin degree of freedom). It behaves as $f_{\mathrm{LM}}=\langle n\rangle$ in the $U \gg \Gamma$ limit, while the small- $U$ dependence is shown in Fig. 5 . None of the contours (iso curves) resembles the sign-reversal curve from Fig. 3. Most notably, the contours in $f_{\mathrm{LM}}$ approach the $n=1$ line with zero slope and do not curve down. Other thermodynamic quantities, such as the characteristic low-energy scale of the problem defined by the temperature where the impurity entropy and the effective moment become small (i.e., the Kondo temperature in the Kondo regime), also correlate with the dependence of $f_{\mathrm{LM}}$ on $U$ and $n$ (not shown). The scattering asymmetry is thus not simply related to the degree of local-moment formation, but requires a calculation of dynamical properties.

We now discuss the relevance of these results to the low- 


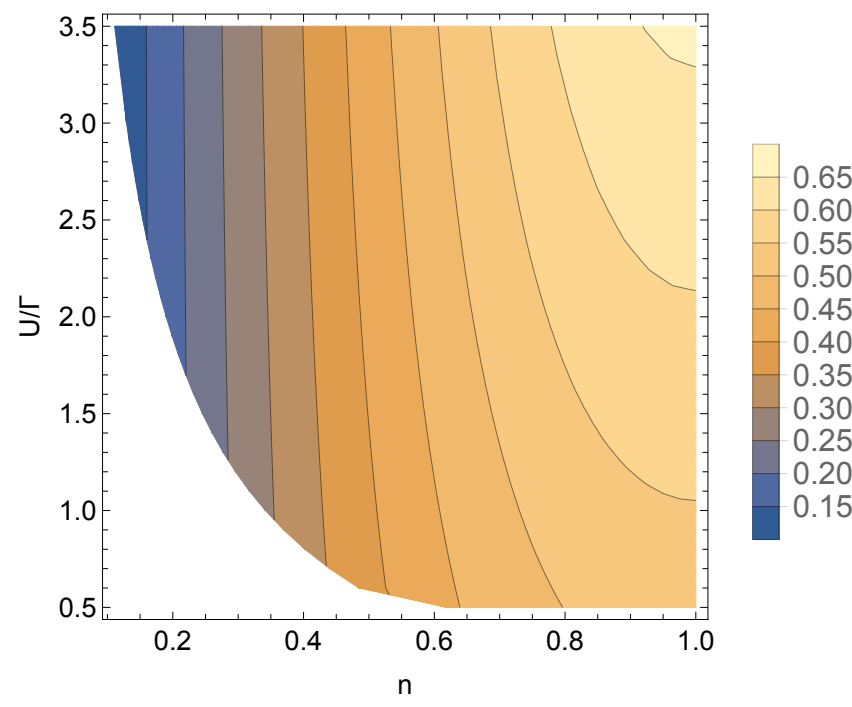

Figure 5. Local-moment fraction $f_{\mathrm{LM}}=\langle n\rangle-2\left\langle n_{\uparrow} n_{\downarrow}\right\rangle$.

temperature thermopower in correlated systems. Two cases need to be distinguished. In quantum dots that are directly described by the SIAM, the thermopower is determined by the asymmetry of the spectral function around the Fermi level, i.e., its Fermi-level slope $\mathrm{e}^{22+24}$. The asymmetry of the particlehole scattering is not important as such, it only enters as one factor that affects the spectral-function slope. The situation is different for bulk systems described by the Hubbard model that map within the dynamical mean field theory (DMFT) approximation to a SIAM with self-consistently defined hybridisation function 25:31. There the thermopower is given by the "leading" term proportional to the Fermi-level slope of the transport function that is "corrected" by a term proportional to the coefficients of the cubic terms in $\operatorname{Im} \Sigma$. This is actually an order 1 correction 2930 , which may become dominant close to half-filling. Due to the DMFT self-consistency, this term has a complex dependence on specific details of the problem (shape of the non-interacting DOS, doping level, strength of the interaction copared to the critical $U_{c 2}$ of the Mott metalto-insulator transition). We plan to present a study of these in a separate publication.

In conclusion, we uncovered a new cross-over line in the phase diagram of the single-impurity Anderson model with flat hybridisation function which corresponds to a change in the scattering dynamics. On one side of this line the impurity behaves as a weakly-renormalized resonant level, on the other side as a magnetic impurity. On the weakly correlated side, the particle-like excitations scatter more strongly than the hole-like excitations, while the opposite is the case on the strongly correlated side. This cross-over might be directly observable in quantum dot experiment ${ }^{32[33}$ provided that the spectral function can be measured in a sufficient energy window so that the reconstruction of the full Green's function $G(\omega)$ is possible via the Kramers-Kronig transformation: assuming that the hybridization function is approximately constant close to the Fermi level, the particle-hole scattering asymmetry can be easily extracted from $\operatorname{Im}\left[G(\omega)^{-1}\right]$. The predicted almost exact particle-hole symmetry of the scattering rate along the cross-over line in the $U-n$ plane should make it an interesting feature to test for in experiments on magnetic adsorbates and quantum dots.

\section{ACKNOWLEDGMENTS}

RŽ acknowledges the support of the Slovenian Research Agency (ARRS) under P1-0044 and J1-7259. The work at UCSC was supported by the US Department of Energy (DOE), Office of Science, Basic Energy Sciences (BES), under Award No. DE-FG02-06ER46319. HRK acknowledges support from the Department of Science and Technology (DST), India.
${ }^{1}$ G.-H. Gweon, B. S. Shastry, and G. D. Gu, "Extremely correlated Fermi liquid description of normal state ARPES in cuprates," Phys. Rev. Lett. 107, 056404 (2011).

2 B. S. Shastry, "Dynamical particle hole asymmetry in cuprate superconductors," Phys. Rev. Lett. 109, 067004 (2012).

3 B. S. Shastry, "Extremely correlated Fermi liquids," Phys. Rev. Lett. 107, 056403 (2011).

${ }^{4}$ B. S. Shastry, E. Perepelitsky, and A. C. Hewson, "Extremely correlated Fermi liquid study of the $U=\infty$ Anderson impurity model," Phys. Rev. B 88, 205108 (2013).

5 B. Horvatić and V. Zlatić, "Perturbation expansion for the asymmetric Anderson hamiltonian," phys. stat. sol. 99, 251 (1980).

6 B. Horvatić and V. Zlatić, "Perturbation expansion for the asymmetric Anderson hamiltonian ii. General asymmetry," phys. stat. sol. 111, 65 (1982).

${ }^{7}$ K. G. Wilson, "The renormalization group: Critical phenomena and the Kondo problem," Rev. Mod. Phys. 47, 773 (1975).

${ }^{8}$ H. R. Krishna-murthy, J. W. Wilkins, and K. G. Wilson, "Renormalization-group approach to the Anderson model of di- lute magnetic alloys. I. Static properties for the symmetric case," Phys. Rev. B 21, 1003 (1980).

${ }^{9}$ H. R. Krishna-murthy, J. W. Wilkins, and K. G. Wilson, "Renormalization-group approach to the Anderson model of dilute magnetic alloys. II. Static properties for the asymmetric case," Phys. Rev. B 21, 1044 (1980).

10 T. A. Costi and A. C. Hewson, "Resistivity cross-over for the nondegenerate Anderson model,” Phil. Mag. B 65, 1165 (1992).

11 Walter Hofstetter, "Generalized numerical renormalization group for dynamical quantities," Phys. Rev. Lett. 85, 1508 (2000).

12 Ralf Bulla, Theo Costi, and Thomas Pruschke, "The numerical renormalization group method for quantum impurity systems," Rev. Mod. Phys. 80, 395 (2008).

13 H. O. Frota and L. N. Oliveira, "Photoemission spectroscopy for the spin-degenerate Anderson model," Phys. Rev. B 33, 7871 (1986).

14 O. Sakai, Y. Shimizu, and T. Kasuya, "Single-particle and magnetic excitation spectra of degenerate Anderson model with finite f-f Coulomb interaction,” J. Phys. Soc. Jpn. 58, 3666 (1989). 
15 T. A. Costi, A. C. Hewson, and V. Zlatić, "Transport coefficients of the Anderson model via the numerical renormalization group," J. Phys.: Condens. Matter 6, 2519 (1994).

${ }^{16}$ R. Bulla, A. C. Hewson, and Th. Pruschke, "Numerical renormalization group calculation for the self-energy of the impurity Anderson model," J. Phys.: Condens. Matter 10, 8365 (1998).

17 V. L. Campo and L. N. Oliveira, "Alternative discretization in the numerical renormalization group," Phys. Rev. B 72, 104432 (2005).

18 Robert Peters, Thomas Pruschke, and Frithjof B. Anders, “A numerical renormalization group approach to Green's functions for quantum impurity models," Phys. Rev. B 74, 245114 (2006).

19 Andreas Weichselbaum and Jan von Delft, "Sum-rule conserving spectral functions from the numerical renormalization group," Phys. Rev. Lett. 99, 076402 (2007).

${ }^{20}$ Rok Žitko and Thomas Pruschke, "Energy resolution and discretization artefacts in the numerical renormalization group," Phys. Rev. B 79, 085106 (2009).

21 A. C. Hewson, The Kondo Problem to Heavy-Fermions (Cambridge University Press, Cambridge, 1993).

${ }^{22}$ R. Scheibner, H. Buhmann, D. Reuter, M. N. Kiselev, and L. W. Molenkamp, "Thermopower of a Kondo spin-correlated quantum dot," Phys. Rev. Lett. 95, 176602 (2005).

${ }^{23}$ T. A. Costi and V. Zlatić, "Thermoelectric transport through strongly correlated quantum dots," Phys. Rev. B 81, 235127 (2010).

${ }^{24}$ S. Andergassen, T. A. Costi, and V. Zlatić, "Mechanism for large thermoelectric power in molecular quantum dots described by the negative- $u$ Anderson model," Phys. Rev. B 84, 241107(R) (2011).

25 Antoine Georges, Gabriel Kotliar, Werner Krauth, and Marcelo J.
Rozenberg, "Dynamical mean-field theory of strongly correlated fermion systems and the limit of infinite dimensions," Rev. Mod. Phys. 68, 13 (1996).

26 B. S. Shastry, "Electrothermal transport coefficients at finite frequencies," Rep. Prog. Phys. 72, 016501 (2009).

27 B. S. Shastry, "New materials for thermoelectric applications: Theory and experiments," (Springer Science + Business Media, Dordrecht, 2013) Chap. Thermopower in Correlated Systems.

${ }^{28}$ K. Haule and G. Kotliar, "Thermoelectrics near the Mott localization-delocalization transition," in Properties and applications of thermoelectric materials, edited by V. Zlatić and A. C. Hewson (Springer Netherlands, Dordrecht, 2009) p. 119.

29 Xiaoyu Deng, Jernej Mravlje, Rok Žitko, Michel Ferrero, Gabriel Kotliar, and Antoine Georges, "How bad metals turn good: spectroscopic signatures of resilient quasiparticles," Phys. Rev. Lett. 110, 086401 (2013).

${ }^{30}$ W. Xu, K. Haule, and G. Kotliar, "Hidden Fermi liquid, scattering rate saturation, and Nernst effect: A dynamical mean-field theory perspective," Phys. Rev. Lett. 111, 036401 (2013).

31 L.-F. Arsenault and A.-M. S. Tremblay, "Transport functions for hypercubic and Bethe lattices," Phys. Rev. B 88, 205109 (2013).

32 D. Goldhaber-Gordon, J. Göres, M. A. Kastner, H. Shtrikman, D. Mahalu, and U. Meirav, "From the Kondo regime to the mixedvalence regime in a single-electron transistor," Phys. Rev. Lett. 81, 5225 (1998).

33 Andrey V. Kretinin, Hadas Shtrikman, David Goldhaber-Gordon, Markus Hanl, Andreas Weichselbaum, Jan von Delft, Theo Costi, and Diana Mahalu, "Spin-1/2 Kondo effect in an InAs nanowire quantum dot: Unitary limit, conductance scaling, and Zeeman splitting," Phys. Rev. B 84, 245316 (2011). 